\title{
Microbial peptide de-coppers mitochondria: implications for Wilson disease
}

\author{
Stephen G. Kaler \\ Section on Translational Neuroscience, Molecular Medicine Branch, Eunice Kennedy Shriver National Institute of Child Health and Human Development, NIH, Bethesda, Maryland, USA.
}

\begin{abstract}
The severe liver pathology of untreated Wilson disease (WD) is associated with massive copper overload caused by mutations in a liver-specific copper-transporting ATPase, ATP7B. While early, presymptomatic detection and chelation with conventional copper-binding molecules enables effective and life-saving treatment, liver transplantation is the sole option currently available for those with advanced disease. In this issue of the $J C I$, Lichtmannegger, Leitzinger, and colleagues delineate the therapeutic effect of methanobactin (MB), a potent bacterial copper-binding protein, at three late stages of disease in a WD rat model. Their results suggest that a formal clinical trial of MB in human subjects with severe hepatic pathology caused by WD would be rational.
\end{abstract}

\section{Wilson disease:}

\section{an overabundance of copper}

Wilson disease (WD) is an autosomal recessive disorder that results in dysfunctional copper transport and accumulation of copper within the liver and brain. WD arises from an inborn error of metabolism and has long enjoyed certain notoriety because, unlike so many other heritable metabolic disorders, WD is considered eminently treatable. WD was clinically described over 100 years ago by S.A.K. Wilson, an American neurologist working in England, and the etiologic connection with copper overload was discerned by the London-based pathologist J.N. Cummings 36 years later $(1,2)$. Oral therapy involving D-penicillamine (D-PA), a small free thiol derived from penicillin, was first conceived in 1955 by J.M. Walshe, a British physician working at Boston City Hospital in Massachusetts (3). Walshe had recognized that the sulfhydryl group of D-PA could make the molecule active as a copper chelator. As once described anecdotally (and modestly) to this commentator, Dr. Walshe prescribed this chemical to a man with WD who was hospitalized at Boston City Hospital, although only after Dr. Walshe had consumed a gram himself! A two-gram supply had been obtained from a Massachusetts Institute of Technology chemist who was a friend of Dr. Walshe's clinical mentor. When Walshe awoke safely in his on-call room the morning after this personal experiment and assessed that "it hadn't killed me," he tested his hypothesis in the patient with WD. A massive increase in urinary copper excretion and clinical improvement were documented in the patient, thereby paving the way for successful treatment of thousands of patients with WD in the decades to follow. Thus, well before the identification of the precise molecular, biochemical, cell biological, and physiological intricacies of WD, copper chelation offered a rational medical treatment (4-7).

\section{Related Article: p. 2721}

Conflict of interest: S.G. Kaler is the First Named Inventor on a patent related to his laboratory's work on Menkes disease, entitled "Codon-optimized reduced-size ATP7A cDNA and uses for treatment of copper transport disorders" (provisional application no. 62/244,594, patent pending).

Reference information: J Clin Invest. 2016;126(7):2412-2414. doi:10.1172/JCI88617.
More advanced stages of WD, which involve severe liver disease or neurological presentations, have, however, been far more difficult to arrest with D-PA or other subsequently developed copper chelators, such as trientine (TETA) and tetrathiomolybdenate (TTM) (8-12). Zinc therapy $(8,11,12)$, an alternative approach based on limiting gastrointestinal absorption of copper via induction of metallothioneins, small cytosolic proteins that sequester copper, has also been unsuccessful in patients with advanced WD. Liver transplantation for WD, which was first performed by T.E. Starzl in Denver in 1975 , remains the only curative treatment available for patients in whom the disease has advanced to fulminant hepatic failure (13). While liver transplantation has been successful in the vast majority of WD patients who require it, this procedure carries significant risks, including complications related to surgery and anesthesia and organ rejection or dysfunction, in addition to the potential side effects of a lifelong immunosuppressant drug regimen.

\section{Methanotroph-derived copper- binding protein shows promise} In this issue, Lichtmannegger, Leitzinger, and colleagues tackle the difficult challenge of medical treatment for advanced WD (14). The authors used a reliable rat model of WD to evaluate the efficacy of a bacterial copper-binding peptide, methanobactin (MB), for the treatment of animals at three progressively worse stages of disease. MB is a well-characterized protein produced by the bacterium Methylosinus trichosporium OB3b, which possesses membrane permeability and exceptionally high avidity for copper, the latter via a unique $\mathrm{N}_{2} \mathrm{~S}_{2}$-binding site (15). Methane-oxidizing bacteria (methanotrophs), such as M. trichosporium, require large quantities of copper to serve as a cofactor for methane monooxygenase activity; therefore, $\mathrm{MB}$ is essential for copper acquisition by these organisms. 


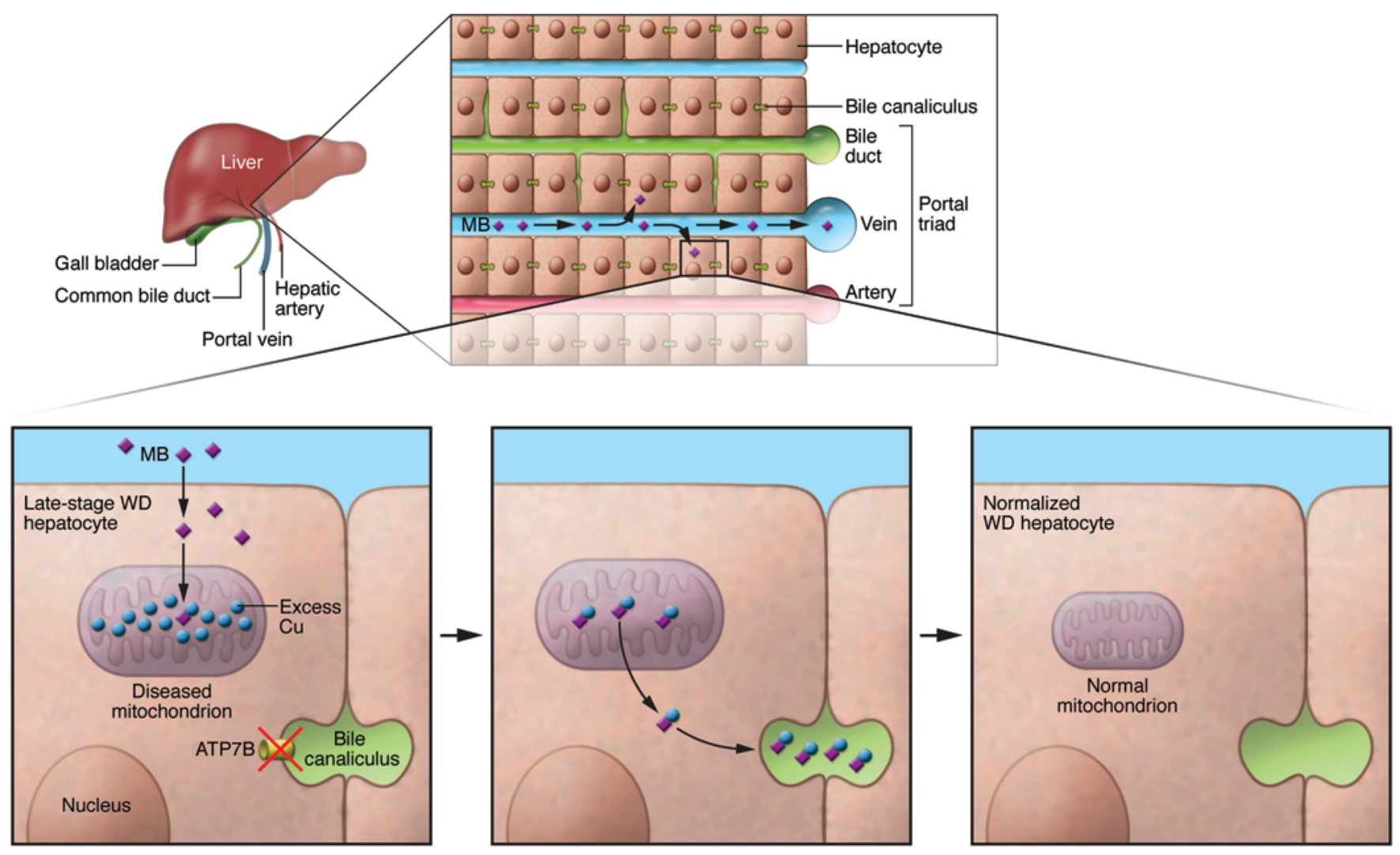

Figure 1. Hepatocyte copper depletion by MB in late-stage WD. Top: Cartoon of the human liver, with details of the portal triad architecture. In late-stage WD, MB (lavender diamonds) administered by i.v. infusion would be taken up by diseased hepatocytes and enter copper-engorged mitochondria (left panel), followed by chelation of copper (blue circles) and exodus from diseased mitochondria of MB-copper complexes destined for biliary excretion (middle panel). The preclinical rat model described by Lichtmannegger, Leitzinger, and colleagues (14) predicts efficient and effective removal of mitochondrial copper by MB, with restoration of normal mitochondrial structure (right panel).

Lichtmannegger, Leitzinger, and colleagues have clearly demonstrated that MB provides superior therapeutic benefit compared with that provided by the standard copper chelators D-PA, TETA, and TTM in the context of severe liver disease in the WD rat model. The authors focused especially on mitochondrial pathology in the diseased rat liver (as well as in cultured hepatocyte-like cells), as the hepatic mitochondrial accumulation of copper that occurs in both WD patients and animal models results in observable ultrastructural abnormalities $(16,17)$. MB treatment impressively and rapidly corrected the massive mitochondrial copper overload, increasing biliary copper excretion at least 10 -fold more than did the alternative chelators (Figure 1). Moreover, MB bound copper much more avidly than did the other chelators tested and easily navigated mitochondrial entry and exit, mirroring the peptide's cell wall permeability in its native bacterium. MB reduced the number of mitochondria showing abnormal vacuolization, dilated cristae, and inner and outer membrane separation and also improved transaminitis in the animals. Most important, MB-treated animals had sustained clinical recovery, including those that were in moribund condition prior to MB administration. These cumulative results suggest that a formal clinical trial of MB in human subjects with severe hepatic pathology due to WD would be rational. The patient population would include those for whom liver transplantation would otherwise be required to preserve life.

\section{Remaining questions and conclusions}

There are several minor caveats and questions that remain to be addressed for this newly proposed clinical application. First, parenteral administration of $\mathrm{MB}$ was required in the current studies, because acid sensitivity and time-dependent tem- perature instability of the product were noted. A modified formulation to enable oral administration, the route for other copper chelators used in WD treatment, would be welcome. Second, MB does not appear to cross blood-brain or blood-cerebrospinal fluid barriers, leaving the urgent need for more effective treatment of the neurological aspects of $\mathrm{WD}$, when present, unmet $(7,9)$. Intrathecal MB administration may be a future consideration for delivery to the CNS. Unfortunately, such an approach cannot be readily assessed, as no current animal models faithfully recapitulate the accumulation of copper in the brain seen in patients with advanced disease. Third, if MB readily enters mitochondria, it seems plausible that this bacterial peptide permeates other intracellular compartments, such as lysosomes, which have recently been shown to perform an important role in normal liver copper homeostasis involving exocytosis (refs. 18, 19). Fourth, while the limited preclinical toxicology 
assessments presented suggest that $\mathrm{MB}$ has a favorable safety profile (14), it is possible that unanticipated side effects could emerge in human subjects. Certain individuals are intolerant of $\mathrm{D}$-PA and experience severe side effects, including nephrotoxicity, hematologic abnormalities, and a distinctive skin rash, elastosis perforans serpiginosa. In some WD patients with neurologic presentations, D-PA treatment induces paradoxical worsening of the clinical phenotype. In the case of $\mathrm{MB}$, any untoward side effects will be weighed in determining whether the risk outweighs the potential benefit in patients with advanced, life-threatening hepatic disease. Fifth, could MB be usefully applied to WD patients with less advanced disease, or in other conditions such as Menkes disease, a state of overall copper deficiency but in which paradoxical and injurious renal copper accumulation occurs (20)?

The detailed studies by Lichtmannegger, Leitzinger, and colleagues (14) suggest the possibility of a welcome addition to the therapeutic armamentarium for patients with WD. As on the wards of Boston City Hospital six decades ago, when John Walshe conceived of copper chelation with D-PA, these investigators now appear poised to test their hypothesis in a clinical trial. Apart from enormous refinements in the protection of human subjects, both examples represent translational research at its best, conveying the exciting potential to improve patient outcomes in a rare and important inherited human illness.

\section{Acknowledgments}

The author is supported by the NIH Intramural Research Program (Z01 HD008768, Z01 HD008892, Z01 HD008927); the NIH Bench-to-Bedside Awards Program; and NIH grants U01 HD079066-01 and U01 HL121842-01A1.

Address correspondence to: Stephen G. Kaler, Porter Neuroscience Research Center II, Building 35, Room 2D-971, 35A Convent Drive, MSC 3754, National Institutes of Health, Bethesda, Maryland 20892-3754, USA. Phone: 301.451.6034; E-mail: kalers@mail.nih.gov.

1. Compston A. Progressive lenticular degeneration: a familial nervous disease associated with cirrhosis of the liver, by S. A. Kinnier Wilson, (From the National Hospital, and the Laboratory of the National Hospital, Queen Square, London) Brain 1912:34;295-509. Brain. 2009;132(pt 8):1997-2001.

2. Cumings JN. The copper and iron content of brain and liver in the normal and in hepato-lenticular degeneration. Brain. 1948;71(pt 4):410-415.

3. Walshe JM. Penicillamine, a new oral therapy for Wilson's disease. Am JMed. 1956;21(4):487-495.

4. Bull PC, Thomas GR, Rommens JM, Forbes JR, Cox DW. The Wilson disease gene is a putative copper transporting P-type ATPase similar to the Menkes gene. Nat Genet. 1993;5(4):327-337.

5. Petrukhin K, et al. Mapping, cloning and genetic characterization of the region containing the Wilson disease gene. Nat Genet. 1993;5(4):338-343.

6. Tanzi RE, et al. The Wilson disease gene is a coppe transporting ATPase with homology to the Menkes disease gene. Nat Genet. 1993;5(4):344-350.

7. Bandmann O, Weiss KH, Kaler SG. Wilson's disease and other neurological copper disorders. Lancet Neurol. 2015;14(1):103-113.
8. Bruha R, et al. Long-term follow-up of Wilson disease: natural history, treatment, mutations analysis and phenotypic correlation. Liver Int . 2011;31(1):83-91.

9. Lorincz MT. Neurologic Wilson's disease. Ann N Y Acad Sci. 2010;1184:173-187.

10. Brewer GJ, et al. Treatment of Wilson disease with ammonium tetrathiomolybdate: IV. Comparison of tetrathiomolybdate and trientine in a double-blind study of treatment of the neurologic presentation of Wilson disease. Arch Neurol. 2006;63(4):521-527.

11. European Association for the Study of the Liver. EASL Clinical Practice Guidelines: Wilson's disease. J Hepatol. 2012;56(3):671-685.

12. Członkowska A, et al. D-penicillamine versus zinc sulfate as first-line therapy for Wilson's disease. Eur J Neurol. 2014;21(4):599-606

13. Sutcliffe RP, et al. Liver transplantation for Wilson's disease: long-term results and quality-of-life assessment. Transplantation. 2003;75(7):1003-1006.

14. Lichtmannegger J, et al. Methanobactin reverses acute liver failure in a rat model of Wilson disease. J Clin Invest. 2016;126(7):2721-2735.

15. Kim HJ, et al. Methanobactin, a copper-acquisition compound from methane-oxidizing bacteria. Science. 2004;305(5690):1612-1615.

16. Sternlieb I. Mitochondrial and fatty changes in hepatocytes of patients with Wilson's disease. Gastroenterology. 1968;55(3):354-367.

17. Roberts EA, Robinson BH, Yang S. Mitochondrial structure and function in the untreated Jackson toxic milk (tx-j) mouse, a model for Wilson disease. Mol Genet Metab. 2008;93(1):54-65.

18. Polishchuk EV, et al. Wilson disease protein ATP7B utilizes lysosomal exocytosis to maintain copper homeostasis. Dev Cell. 2014;29(6):686-700.

19. Peña K, Coblenz J, Kiselyov K. Brief exposure to copper activates lysosomal exocytosis. Cell Calcium. 2015;57(4):257-262.

20. Kaler SG, et al. Neonatal diagnosis and treatment of Menkes disease. $N$ Engl J Med. 2008;358(6):605-614. 